\title{
A Wireless Sensor System for Identifying Bed Exits for Hospitalized Older People
}

\author{
Sunil Kudupudi, J. Mohana
}

\begin{abstract}
Falls of older people in hospitals are common, so there should me techniques in order to reduce these falls. Pressure sensor systems were used for the detection of these falls, which resulted in a larger number of false alarms. Wireless sensors became more advanced in current scenario and using these wireless sensors falls can be prevented mostly. Unlike pressure sensors usage which needs a constant maintainance of the system wireless sensor system is more better in many ways. The type of design which can monitor health condition and analyze the movement and give an alert if patient is moving out of his bed going wrong and we can transmit data wirelessly anywhere by using GSM technology.
\end{abstract}

\section{INTRODUCTION}

Falls are real causes in more seasoned individuals for wounds which can be anticipated. Aside from physical damage and included monetary weight from related costs, falls cause mental injury, for example, dread, loss of certainty, nervousness and discouragement, which thusly sway on a more established individual's autonomy cautions. Notwithstanding the poor execution of weight mats put over the seat or sleeping cushion, these sensors require steady support, for example, cleaning and purification as weight mats are probably going to be in contact with body liquids, and hence expanding the remaining task at hand of staff. In addition, discernable alerts result in 'clamor' that bothers patients, particularly those with intellectual debilitation. Different ponders have likewise utilized video pictures for anticipating falls However, past examine has demonstrated the indication of protection worries with the utilization of cameras in more established individuals' living condition. The investigation in among network staying more seasoned individuals reports that cameras raised more prominent protection worries than different advances, notwithstanding when techniques for extricating outlines were set up to safeguard security. Further, the power of vision based acknowledgment methods can be tested because of various reasons, for example, evolving enlightenment, mess, dynamic foundations, impediments and, in a clinic setting, various individuals, patients or guests in a patients ward room.Wearable sensors for falls prevention system can provide new opportunities for individualized monitoring of patients where human motion data can be collected and transmitted in real time for analysis. In addition, as sensors are decreasing in size and power consumption, the inclusion of multiple sensors can further extract physiological signals of interest along with motion data. Most studies using wearable sensors that target older people for the recognition of unassisted bed exits.

\section{LITERATURE SURVEY}

1.To investigate distributed emergency clinic fall counteractive action projects to decide if there is any impact on fall rates. To survey the methodological nature of those projects and the scope of mediations utilized. To give bearings to additionally inquire about. Methodical survey of distributed emergency clinic fall counteractive action programs. Meta-examination. Watchword hunts of Medline, CINAHL, monographs, and optional references. All papers were incorporated that depicted fall rates previously and amid mediation. Hazard proportions and 95\% Confidence Intervals $(95 \% \mathrm{CI})$ were assessed and irregular impacts meta-investigation utilized. Begg's test was connected to recognize conceivable distribution predisposition. Separate meta-investigation relapses were performed to decide if singular segments of multifaceted intercessions were powerful.

2.Wireless body sensor organize (WBSN) innovations are viewed as one of pulling in research territories in software engineering. At the point when joined with the human services application, it gives high esteem innovation of far reaching human services checking arrangement in extraordinary circumstances including high elevation or hazardous situation empowering the ground controller to screen remote pilots or seismic tremor exploited people continuously by mix of remote sensors and sensor systems.

3.Accelerometers may help spare the lives of the individuals who are older or who experience issues standing. These touchy accelerometers are utilized in various fall discovery gadgets. They sense when somebody has abruptly fallen by deciding the adjustment in their speed and toward the path, they are moving. In the event that the gadget verifies that the qualities for these two factors fall into the peril class, it will naturally send a fall caution and call for help

\footnotetext{
Revised Manuscript Received on September 10, 2019.

Sunil Kudupudi, (B.Tech), Department of electronics and communication engineering, Saveetha school of engineering, Chennai, Tamilnadu, India.

Ms. J.Mohana, (Assistant professor), Department of electronics and communication engineering, Saveetha school of engineering, Chennai,
} Tamilnadu, India. 


\section{A Wireless Sensor System For Identifying Bed Exits For Hospitalized Older People}

\section{METHODOLOGY}

BLOCK DIAGRAM

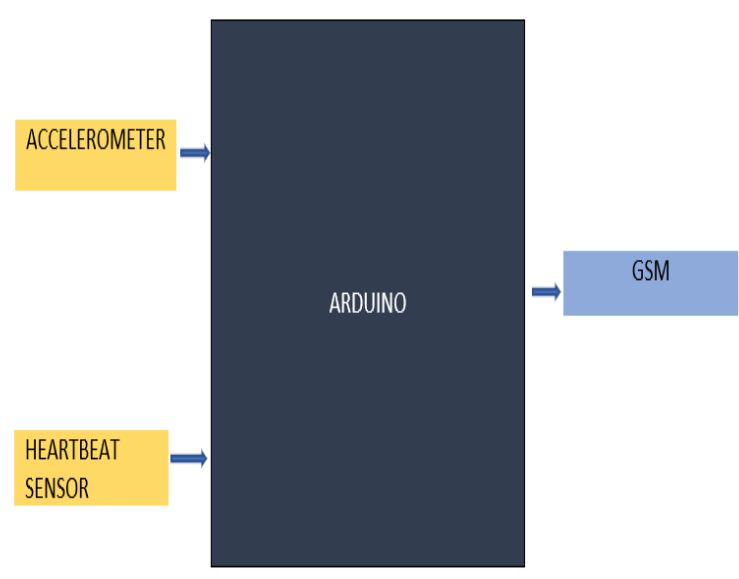

TOOLS USED

\section{1) Arduino}

Used for the conversion of accelerometer analog readings to digital readings and also to read the heartbeat sensor digital readings and acts as an heart of this project.

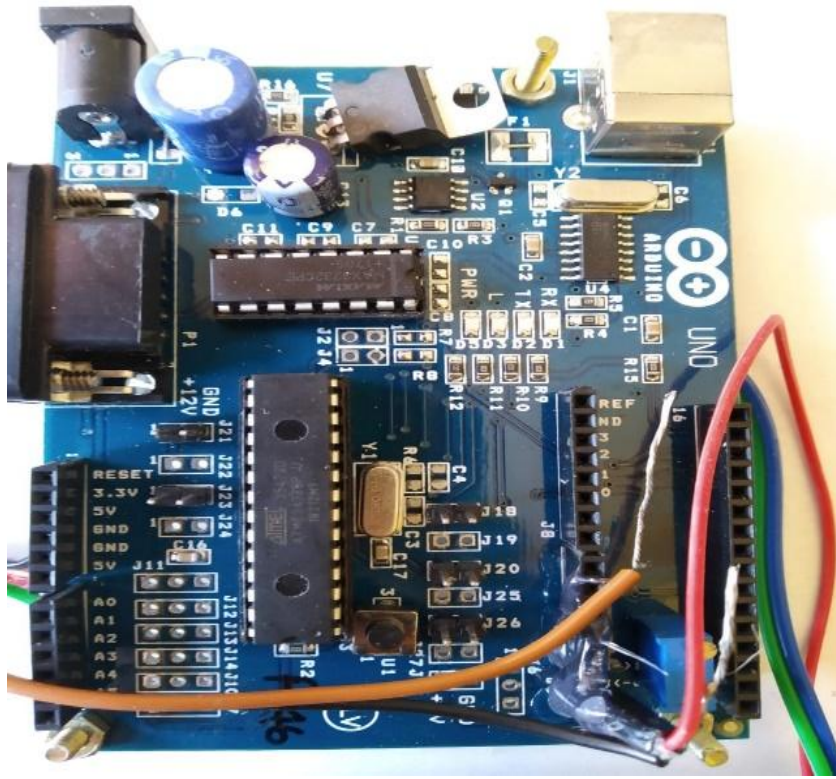

2) MEM sensor

It constantly reads the movement of the patient.

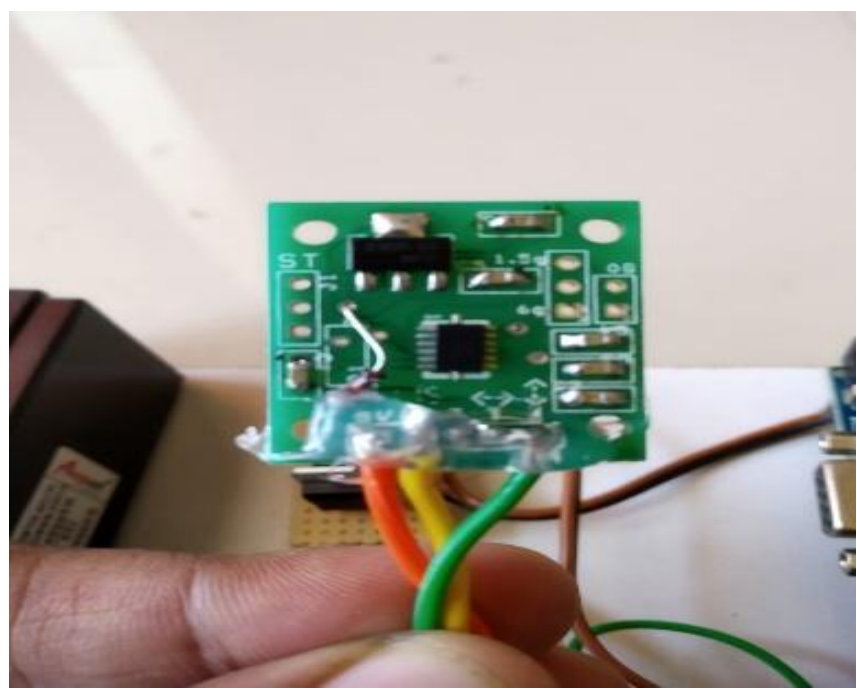

3) Heart beat sensor

It reads the heartbeat of the patient. It can directly read the digital signals of the heart.

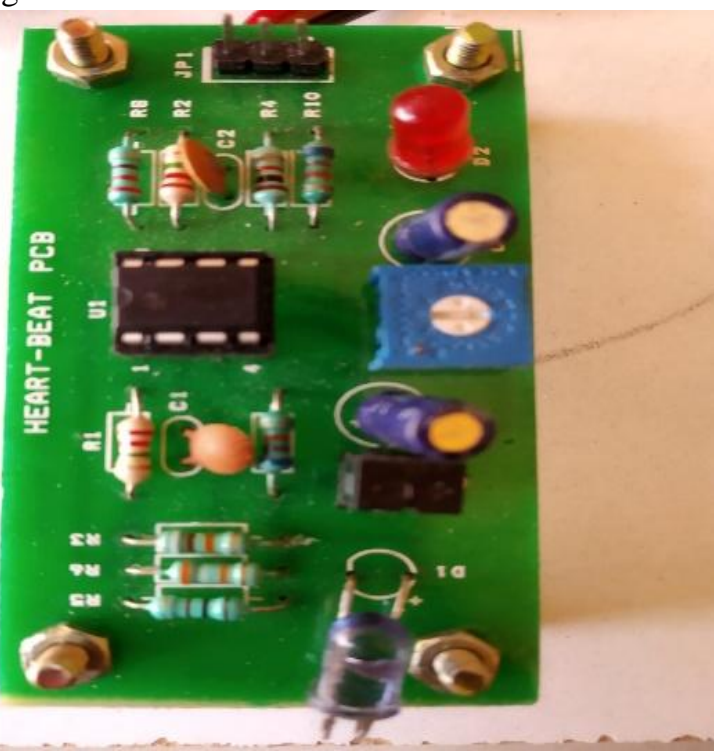

\section{4) $G S M$}

Used for transmitting the information to wherever the alert needs to be send.

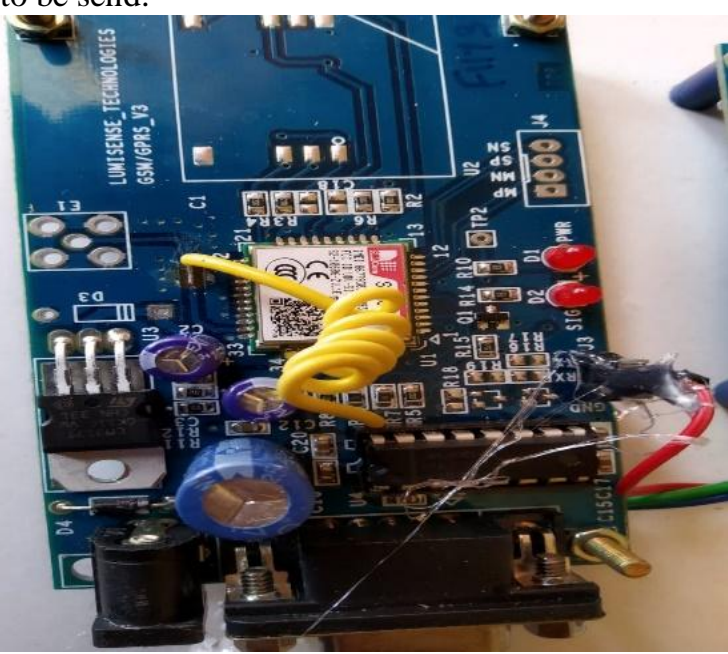

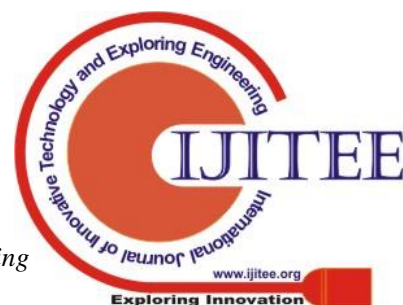




\section{WORKING}

An Arduino setup with inputs including triaxial accelerometer and a heartbeat sensor which gives an alert about the heartbeat all time are inputs to the Arduino and a GSM is used for the wireless transfer for alerts if the patient is attempting to move or if heartbeat is high. The triaxial accelerometer analog data is converted into digital by ADC pins in the Arduino and these signals are to be analysed using an embedded software and using an algorithm the movement of the patient can be noticed and if a patient moves, the readings of accelerometervaries and an alert is send to the staff in the hospital and the patient will be assisted and in this way we can reduce the falls in hospitals.

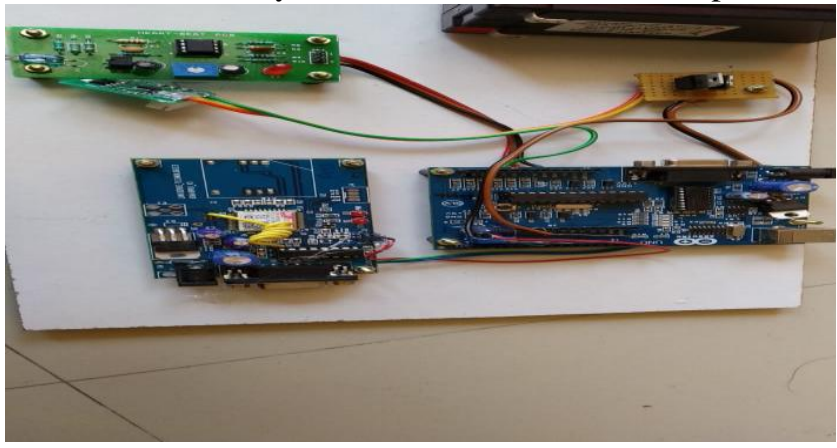

Accelerometers may help spare the lives of the individuals who are older or who experience issues standing. These touchy accelerometers are utilized in various fall discovery gadgets. They sense when somebody has abruptly fallen by deciding the adjustment in their speed and toward the path, they are moving. In the event that the gadget verifies that the qualities for these two factors fall into the peril class, it will naturally send a fall caution and call for help.

\section{CODE}

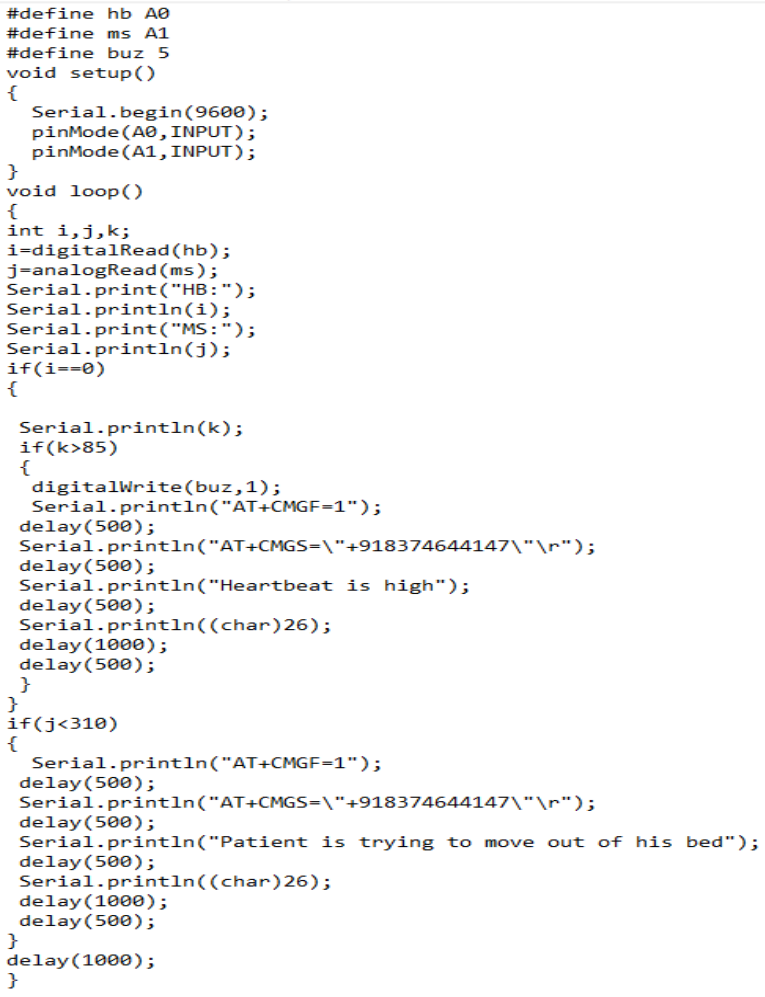

Using this wireless sensor technology number of falls can be reduced in older people and the heart beat alert helps the management in saving lives which are lost without the knowledge of doctors and staff. Wireless sensor technology helps to develop the medical applications in a vey sophisticated manner and health of the patient is monitored continuously. Evolution of wireless sensor technology will make monitoring and maintaining the patients' health easier.

\section{REFERENCES}

1. https://www.researchgate.net/publication/283551552_Re search_challenges_of_IEEE_802156_standard_for_wirel ess_body_area_networks

2. https://dl.acm.org/citation.cfm?id=1038146

3. Rich DeVaul, Michael Sung, Jonathan Gips , Alex Sandy Pentland, MIThril 2003: Applications and Architecture, Proceedings of the 7th IEEE International

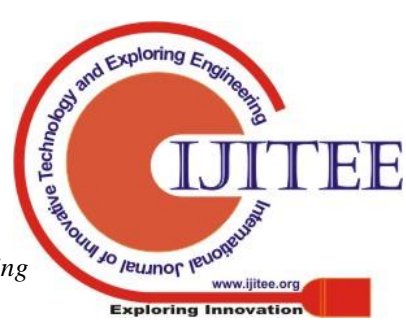


Symposium on Wearable Computers, p.4, October 21-23, 2003

4. Eric Dishman, Inventing Wellness Systems for Aging in Place, Computer, v.37 n.5, p.34-41, May 2004 [doi>10.1109/MC.2004.1297237]

5. https://www.researchgate.net/publication/318645241_Tr affic-

Aware_Medium_Access_Control_Protocol_for_Wireless _Body_Area_Networks

6. https://ieeexplore.ieee.org/document/6739368

7. Australian Commission on Safety and Quality in Healthcare. Preventing Falls and Harm From Falls in Older People: Best Practice Guidelines for Australian Hospitals; 2009.

8. Centers for Disease Control and Prevention. Cost of falls among older adults; 2015. Available from: http://www.webcitation.org/6hanayMoC.

9. Hitcho EB, Krauss MJ, Birge S, Claiborne Dunagan W, Fischer I, Johnson S, et al. Characteristics and circumstances of falls in a hospital setting. J Gen Intern Med. 2004;19(7):732-739. 10.1111/j.15251497.2004.30387.x [PMC free article] [PubMed] [CrossRef], 